\title{
Damage Detection by Template Matching of Scattered Waves
}

\author{
by Anthony Massari," Robert W. Clayton, and Monica Kohler
}

\begin{abstract}
A method based on template matching is presented to detect and locate damage in buildings following severe shaking by an earthquake. The templates are constructed by finite-element simulations of a suite of damage scenarios, with the solutions evaluated at the location (and orientation) of each sensor in the structure. The damage detection is carried out by cross-correlating the templates with recordings acquired from earthquakes. A dense distributed network of sensors is important for detecting anomalies in the presence of ambient noise. The cross correlation of the templates with themselves provides a measure of the resolution of the damage location.
\end{abstract}

\section{Introduction}

One of the important tasks in building resilient structures is monitoring the state of health of the building after it has been strongly shaken by an earthquake. Major damage is usually apparent by a simple visual inspection, but more subtle damage, such as broken moment welds, may leave the building vulnerable to another earthquake but otherwise have no apparent manifestation (Miller, 1998). Inspecting for this type of damage is difficult, expensive, time consuming, and may significantly slow the recovery of a city. Two recent innovations may contribute to a solution to this problem. The first is the use of network of sensors, such as the Community Seismic Network (CSN; Kohler et al., 2013, 2014; Clayton et al., 2015), that can significantly increase the density of observations of internal motion in the building before, during, and after an earthquake. The second is the widespread use of models of structures during the design phase. These are often in the form of finite-element models that can be used to predict building dynamics due to ground shaking.

Detecting damage is often carried out by looking for changes in the building performance before and after the earthquake. An overview of damage-detection methods is presented in Farrar and Worden (2007) and Qiao and Esmaeily (2011). One approach is to look at changes in the modal properties of a building by looking at frequency shifts in the building modes (e.g., Todorovska and Trifunac, 2007). For this to be effective in localizing the damage, higher-order modes of the building need to be used. However, the modal properties of the building can also be affected by nonearthquake factors, such as soil conditions, age of the building, and weather. In a recent study of mode frequency and shapes of a 20-story building based on four nondamaging earthquakes, Wen and Kalkan (2017) show that there is considerable variation between earthquakes. This indicates that this approach might be difficult to apply to damage detection and

\footnotetext{
*Now at Department of Civil, Environmental and Geodetic Engineering, Ohio State University, Columbus, Ohio 43201; Massari.8@osu.edu.
}

localization. Daily variations in the modal frequencies on the order of $1 \%$ that are highly correlated with humidity and temperature have been shown for a 20 -story building (Mordret et al., 2017).

Another approach is to look for the change in properties in the time domain. For example, damage to a structural component may change the aggregate stiffness in a portion of the building that in turn will lead to a change in the velocity of waves that travel through the zone (e.g., Todorovska and Trifunac, 2008). With an array of sensors, this change in velocity may be detected, and tomographic algorithms can be used to locate the damage zone. The velocity changes will likely be very small, and a dense array will be needed to detect them. The use of reverse-time wave-propagation methods that apply a full waveform analysis to the problem described by Heckman (2014) and Heckman et al. (2011) has been applied to laboratory models. The above-mentioned study by Wen and Kalkan (2017) also shows that the level of velocity anomalies varies considerably for the suite of nondamaging earthquakes. There are also weather-related changes in the apparent velocity on a daily and monthly scale (Mordret et al., 2017).

In this article, we consider a different approach that is based on scattered waves. Similar to the previous two approaches, this method depends on a change in the aggregate stiffness properties, but in this case the phenomenon being exploited is the wave that is scattered or reflected from this change. The time-domain methods mentioned above look for changes in the waves transmitted through a damaged zone, whereas this approach uses waves that are reflected from it. The new reflected or scattered waves do not exist in an undamaged building, and hence this method is focused on detection, not measuring changes.

The scattered waves mentioned above are likely to be very small compared to the overall response but will propagate through the building in a predictable manner. Detecting them is not unlike finding and locating earthquakes, and a 
recently developed technique for doing this is template matching (Shelly et al., 2007; Huang and Beroza, 2015; Yoon et al., 2015). With this method, a template is used to capture all the details of wave propagation, either using a small recorded earthquake or using a set of synthetics based on a model. With a template for every potential source location, the detection and location is done by cross-correlating the data recorded at several sensors with the templates. Correlations that exceed a prescribed threshold are declared to be detections of damage located at the source point of the template. The level of detectability is determined by the level at which the correlation output exceeds the correlation of ambient noise, and the uniqueness of location is determined by cross-correlating the templates with themselves. The effectiveness of the template method will depend on the detection of the scattered waves. This will generally increase with more sensors in the building but will also depend on where the sensors are located relative to the damage zone. These are the same issues that arise in designing a seismic network for earthquake location.

In this article, we introduce template matching as a possible solution to the problem of damage detection and localization. The technique is applied to two quite different buildings that are monitored by dense arrays of accelerometers that are part of the CSN (Kohler et al., 2014; Clayton et al., 2015), and for which we have detailed structural engineering plans to allow a detailed finite-element model (ETABS, Computers and Structures, Inc.) to be developed. The example motions are simulated with this code because no damaging earthquakes have been recorded in these building while the dense array was in place. The models allow a set of templates to be constructed for various damage scenarios. The CSN sensors provide realistic measurements of ambient noise to compare to the detections.

\section{Scattered Waves}

The damage technique proposed in this article is based on reflected or scattered waves. A simple relation that is used to describe first-order scattering is the Born approximation

$$
G=G_{r}+G_{r} V G_{r},
$$

in which $G$ is the Green's function that describes the wave propagation from a source point to a sensor in the damaged building, and $G_{r}$ is the same thing in the undamaged building. The scattering potential $V$ contains the impedance changes that generate the scattered waves. It is formally defined as the difference in the wave operators between the damaged and undamaged medium (building). In general, it will depend on frequency and incidence angle, but for the purposes of this article, we will take the scattering potential to contain the location of the damage and its floor level as manifested by the apparent change in impedance. Equation (1) is an approximation that neglects multiple interactions with the damaged zone that should be accurate if the impedance changes caused by damage are small. Multiples unrelated to damage are included in the reference Green's functions.

The leading term in the Born approximation is the direct wave and contains no information about the scattered wave. For a quasi 1D problem, such as with tall buildings, this is also true of the first $G_{r}$ in the second term on the right side of equation (1). Because direct waves are usually larger than the scattered wave, we attempt to remove or at least minimize them by subtracting the response of a nondamaging earthquake, generated using the model of the building. With the direct wave removed, the data is described by

$$
D=S * V G_{r},
$$

in which $S$ is the source-time function and $D$ is the observed data with the direct wave removed. Here, $V(x, y, z)$ acts as the source of the scattered wave generated by the damage zone. The challenge is to find regions in $V$ that are above a threshold value from measurements $D$. Estimating the source can be done using previously recorded events or by deconvolving the data with the recording on the lowest floor.

\section{Construction of the Templates}

To detect signals such as the one described by equation (2) in the presence of noise, we propose using template matching (also known as pattern matching) (Shelly et al., 2007; Yoon et al., 2015). To do this, we make a set of templates of the form

$$
T_{i}=P_{i} G_{r},
$$

in which $T_{i}$ is the $i$ th template and $P_{i}$ is the $i$ th damage scenario or location of interest. This is usually represented as the reduced capacity of a particular structure member (e.g., brace connection), or it can be aggregate damage on a floor. In either case, we use a directed force to represent the damage. The $G_{r}$ term in equation (3) propagates the waves to the sensors in the building and in our case is calculated using a linear finite-element model of the building (ETABS model).

To utilize the templates, we simply cross-correlate the modified data ( $D$ in equation 2 ) with each template. That is, we construct a correlation vector

$$
C_{i}=T_{i} * D
$$

The damaged points in the building should correspond to the maxima in the correlation vector $C_{i}$. Several damaged areas will generate multiple maxima in $C_{i}$. The level of damage is proportional to the magnitude of $C_{i}$. The resolution of damage at a test point is given by a resolution matrix

$$
R_{i j}=C_{i} * C_{j}
$$




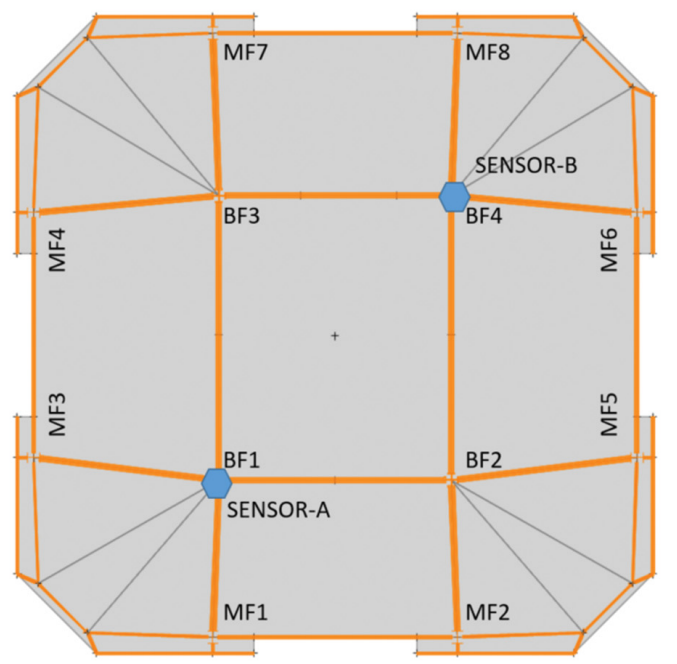

Figure 1. The 52-story building example. Proxy damage locations (BF1-BF4 and MF1-MF8, in which BF is brace frame and MF is moment frame) as well as receiver locations (SENSOR-A and SENSOR-B) are shown. Each floor has a total of 12 potential damage locations, thereby necessitating 588 unique templates. Sensor locations are based on actual layout of existing and anticipated expanded network of sensors in the existing building.

that shows how well one test point can be distinguished from the others. Examples of the templates and resolution matrices are shown in the next sections.

\section{Models of the Buildings}

In this study, we consider two buildings: a 52-story building in downtown Los Angeles, California, and a nine-story building on the campus of the Jet Propulsion Laboratory (JPL) in Pasadena, California. For each building, a model is constructed from the structural engineering drawings, from which the structural elements for each floor are used as input to construct a complex, linear model. This model does an excellent job of simulating the linear motions of the building based on modeling small earthquakes (Clayton et al., 2015).

The model has approximately 30,000 degrees-offreedom, with masses lumped at the story levels with rigid diaphragm constraints. This effectively reduces the dynamic degrees of freedom to only include the story-level responses and negates any inplane relative motion.

The model is fully linear; however, damage is expected to occur due to some nonlinearity in the building elements when they are driven beyond their capacity. Rather than focusing on capturing the damage as it happens during any nonlinear excursion, we use a damaged linear model reflecting the postevent reduced stiffness and seek to observe variations in anticipated wave characteristics. Damage is induced by either reducing stiffness of a section using linear property modifiers or simulating failed connections by removing fixity from selected member locations.

\section{Example of a 52-Story Building}

The first example we show is a tall building in downtown Los Angeles that is shown in Figure 1. This is a 52-story $(+5$ basement levels) dual-system building with an interior concentrically braced core surrounded by outrigger moment frames. The building was constructed in 1988 and is used exclusively as an office building. Examples of earthquake and ambient noise waves propagating in this building, as recorded by the dense CSN network, are shown in Clayton et al. (2015) and Kohler et al. (2016). The performance of the building, including modal frequencies, velocity, and damping variations is described in Celebi et al. (2016) and Kohler et al. (2016).

Templates for the 52-story building were developed for a series of plausible damage locations selected at the connections of braces, columns, and beams. Each floor has 12 potential templates (eight moment-frame connections and four brace-frame connections). For all measured elevated floor levels (52 floors), this leads to a total of 588 damage locations (see Fig. 1). Each damage location has a unique template. The response of a select few of these templates is shown graphically in Figure 2. The templates shown in this figure are shifted in time to match the arrival time of the upgoing wave.

The resolution of one particular damage scenario from the others can be shown by the correlation of the templates with each other. Figure 3 shows the correlation of all of the brace-frame-damage proxy templates for the BF4 location shown in Figure 1 to each other. The banded nature of this plot indicates a high level of orthogonal behavior between any one template and the others.

For this building, five separate damage scenarios are selected and implemented by performing stiffness reduction of brace objects in multiple locations (area reduced to $75 \%$ of the original value). Table 1 summarizes each of these damage scenarios. For each damage scenario, a Gaussian input in displacement was imparted at the base of the structure.

Each damage scenario takes the template information described in Figures 1 and 2 and correlates each to all the receivers located throughout the structure. All 12 damage template correlations of a given floor are numerically integrated together to describe a total floor-level correlation.

The strength of the correlation is dependent on the number and location of sensors used throughout the building. We experimented with several sensor configurations, and in particular we show the results when there are sensors at (1) every floor and (2) every 2nd, 5th, 10th, and 20th floor. The relative strength of the correlation is also dependent on the signal-to-noise ratio of the structure, and in this case we 

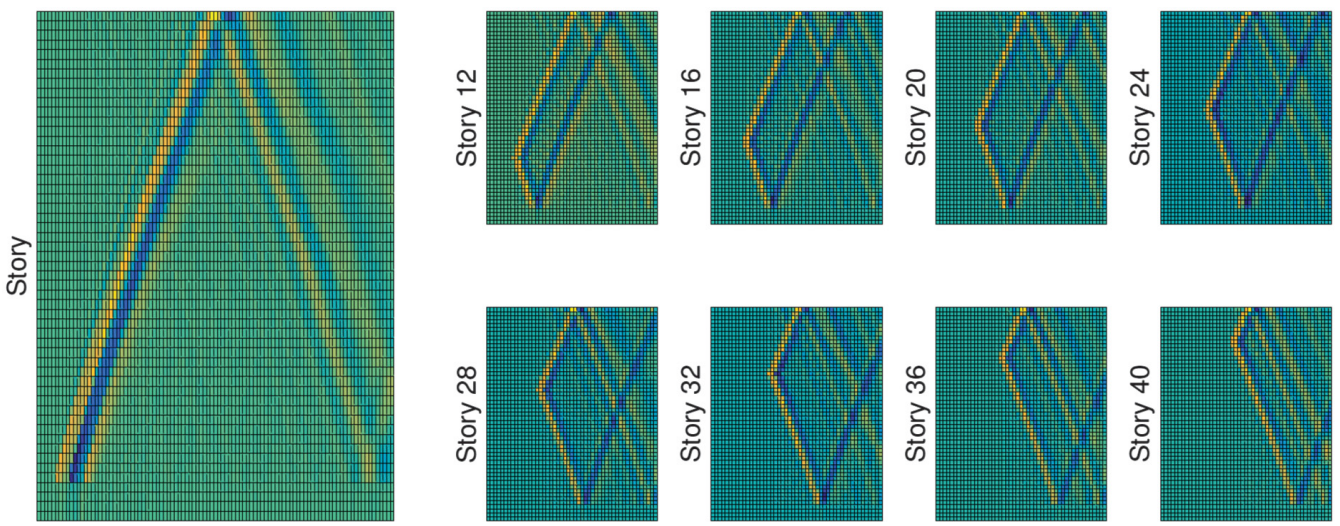

Figure 2. The 52-story building templates showing overall response to impulse functions shifted in time to match the wavespeed of the structure. All template durations shown are $3 \mathrm{~s}$ long (50\% of the building period).

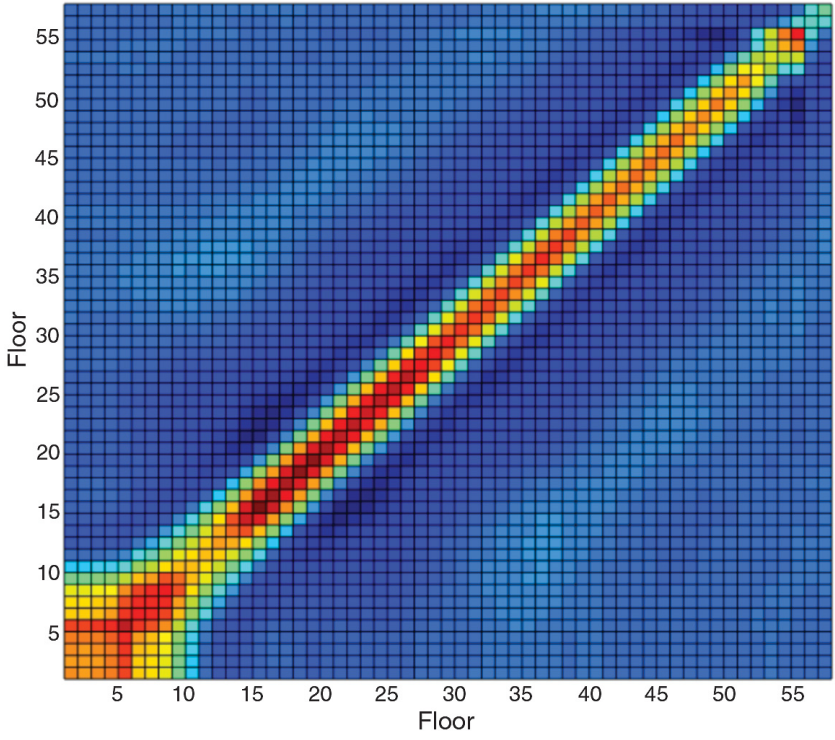

Figure 3. The Impulse orthogonality matrix. The 52-story building template cross correlations are shown. The diagonal nature of the matrix shows the orthogonal nature of one template relative to another. The uniqueness of one template to another is what allows for isolation of damage on a story by story basis in this structure.

Table 1

Damage Scenarios Applied to the 52-Story Building

\begin{tabular}{cc}
\hline Scenario & Damage Applied \\
\hline 1 & Level 35 \\
2 & Levels 35 and 20 \\
3 & Levels $33-35$ \\
4 & Level 20 \\
5 & Level 25 \\
\hline
\end{tabular}

Each damage scenario corresponds to a $25 \%$ reduction in the area of all braces on the floors noted. use the actual noise measurements from the CSN sensors in the building.

To summarize these results, Figure 4 demonstrates graphically what the analysis results look like for damage scenario 1 (Table 1) with and without noise. Each of the subsequent graphs shows the other damage scenarios described in Table 1 and the effect of reducing the number of sensors used in the correlations. Here, it is observed that additional sensors increase the amplitude of the correlations overall. Also, the effect of noise being added to the system reduces the clarity in the image, thereby indicating a signal-to-noise sensitivity to the method. A comparison of scenarios 1 and 3 shows that damage on sequential floors broadens the correlation peak, as would be expected. Scenario 2 shows the level to which separated damage can be distinguished.

\section{Example of a Nine-Story Building}

A similar study to the 52-story example was conducted for a nine-story (+1 basement level) building on National Aeronautics and Space Administration's JPL campus in Pasadena, California. In this example, we explore deeper (higher spatial resolution) into the correlations in a particular floor level. Instead of performing entire floor-level integration of all damage locations, as with the 52-story example, we examine the interaction of multiple damage locations or scenarios on each floor.

This building, constructed in 1961, offers distinct trussframe system characteristics that make it uniquely interesting for studying the damage signature of local buckling of individual truss components. In the north-south direction, lateral loads are resisted by concrete-encased steel columns that act as rigid frames and the welded steel trussed floor girders that are bolted to the steel column sections. In the east-west direction, loads are resisted by columns and the trussed spandrel girders. Additional stiffness is added in the lower floors by earth retaining walls in the basement and the first story 
(a) All damage scenarios with skipping floors no added noise

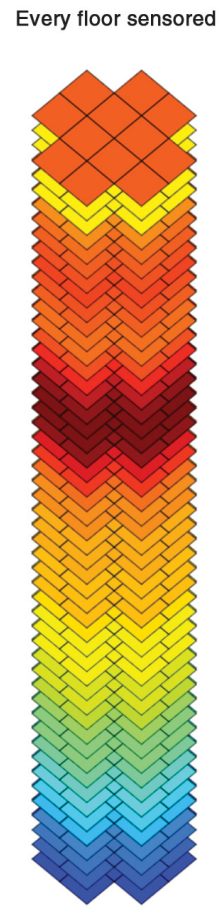

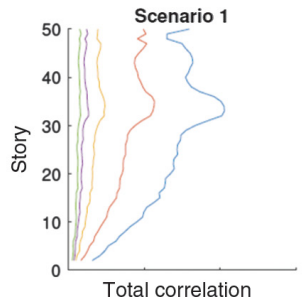
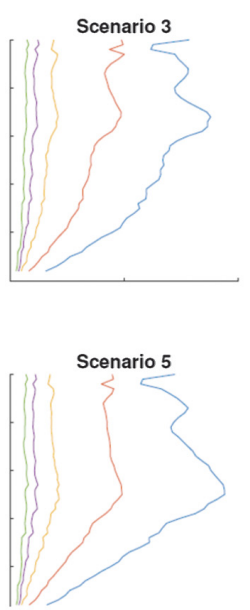

(b)
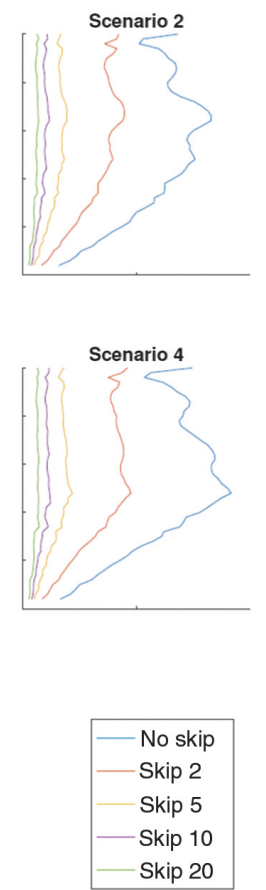

Figure 4. All damage scenarios with varied levels of sensors in the correlations are shown (using either all stories or skipping every 2, 5, 10 , or 20 stories). The subplots show the correlations for the various scenarios, with the vertical axis being floor from 1 to 50 and the horizontal axis being correlation from 0 to 1 . (a) The case of no noise added to the signal. (b) Adding a white-noise amplitude of $10 \%$ of the maximum amplitude of the peak signal on any floor.

(Wood, 1972). The trusses offer an atypical damage condition to assess when compared with standard-moment frames. The building's planar aspect ratio is approximately $6: 1$, making it particularly stiff in the east-west direction, in turn making it more susceptible to torsional excitation or amplification from north to south input.

The unique aspect ratios and torsional properties of the building create a pronounced correlation between collinear source locations in the $Y$-direction. For any north-south frame line (Fig. 5), we see strong correlation in the $Y$-direction (north-south) for any collinear set of impulses (e.g., S2 and N2); however, lower correlation is observed when impulsive source functions are measured on collinear gridlines

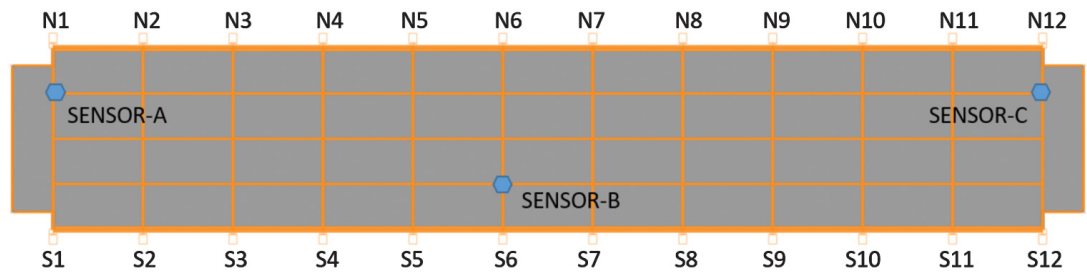

Figure 5. The nine-story building proxy damage locations (N1-N12 and S1-S12), as well as receiver locations (SENSOR-A, SENSOR-B, and SENSOR-C), are shown. North is up. Each floor has a total of 24 potential damage locations, necessitating a total of 192 templates to allow for isolation. (e.g., S2 and N11). Alternatively, in the $X$-direction (eastwest), there is far less variation in the template correlations relative to the location within the floor where the source function originates (see Fig. 6, $X$-correlations). This indicates that isolating a particular location in a floor plate can be more effective if the damage affects stiffness in the northsouth direction as opposed to east-west that will limit our ability to locate damage with an intrafloor resolution.

For the nine-story building, different damage scenario types were used compared to the 52-story building. Damage was isolated to the space between floors six and seven and only involved a single-element stiffness reduction. Truss beams were used to develop the moment frames in the building, which allows for investigation of the damage potential of truss-connection failure. Each damage scenario consisted of reducing the bottom chord of any particular truss connection to $1 \%$ of the initial area representing a severe connection failure.

Figure 7 aggregates the correlations of all of the damage scenarios in Table 2. Observe that the highest amplitude in correlation is spread over floors 6-8 for all the damage scenarios. Comparing damage 
(a)

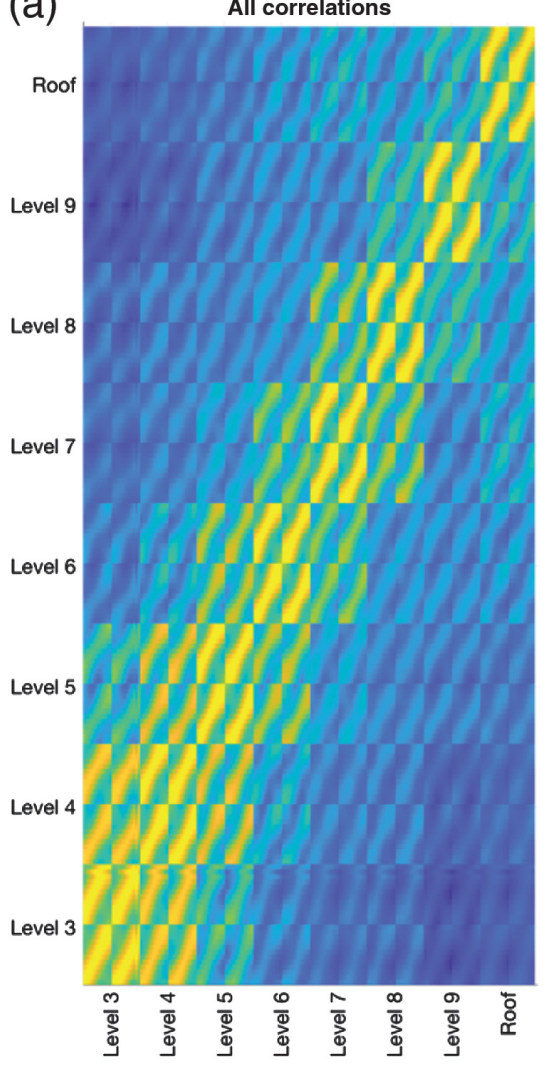

(b)

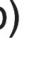

Roo

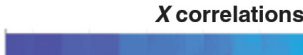

(c)

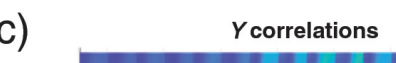

Level

Level 8

Level

Level 6

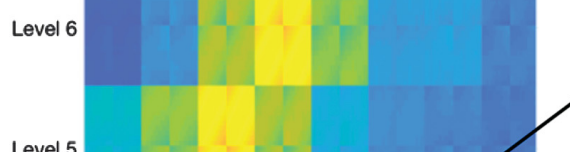

Level 5

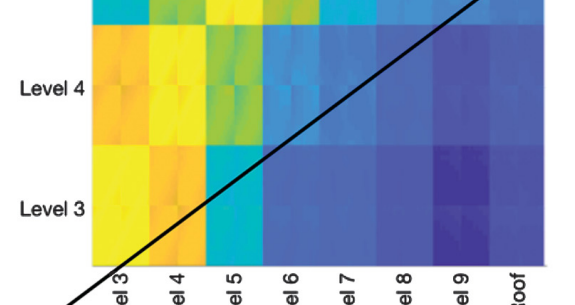

Level 5

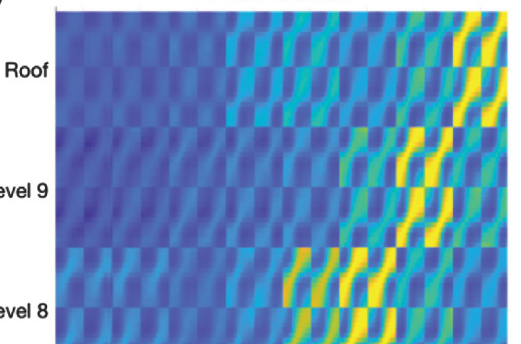

Level 3
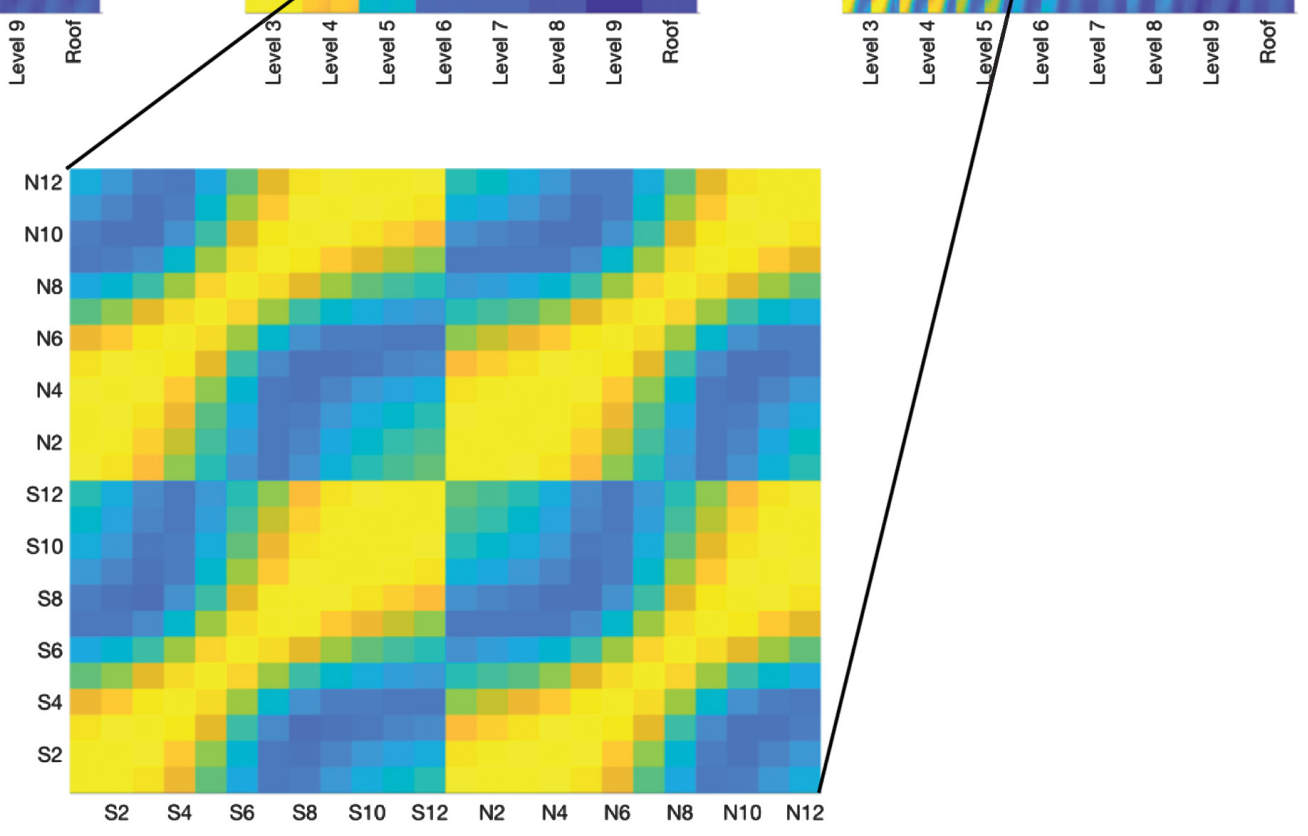

Figure 6. (a) The nine-story building template correlations showing the orthogonal nature of one template to another. (b) The $X$ (eastwest) correlations show the lack of orthogonality of the templates on a floor-by-floor basis. (c) The blowup of level seven correlations in the $Y$ (north-south) correlations shows the banded nature of two concurrent templates on a particular gridline. This indicates the orthogonality of adjacent gridlines but the strong coupling between common $Y$-gridline templates. Note that the roof is the 10th floor.

scenario 2 to scenario 4 (Table 2), the relative amplitude of the correlation increases drastically, due to the torsional effect produced by the eccentricity of the reflected wave relative to the center of rigidity of the structure. This can be further isolated only by correlating in either direction for each damage scenario (see Fig. 8). Observe the lack of amplitude in any directional correlation for which damage was imparted (Fig. 8). This type of directional correlation information can be used to determine damage orientation.

\section{Discussion}

The examples described here indicate that there is potential for the template-matching method to detect and 

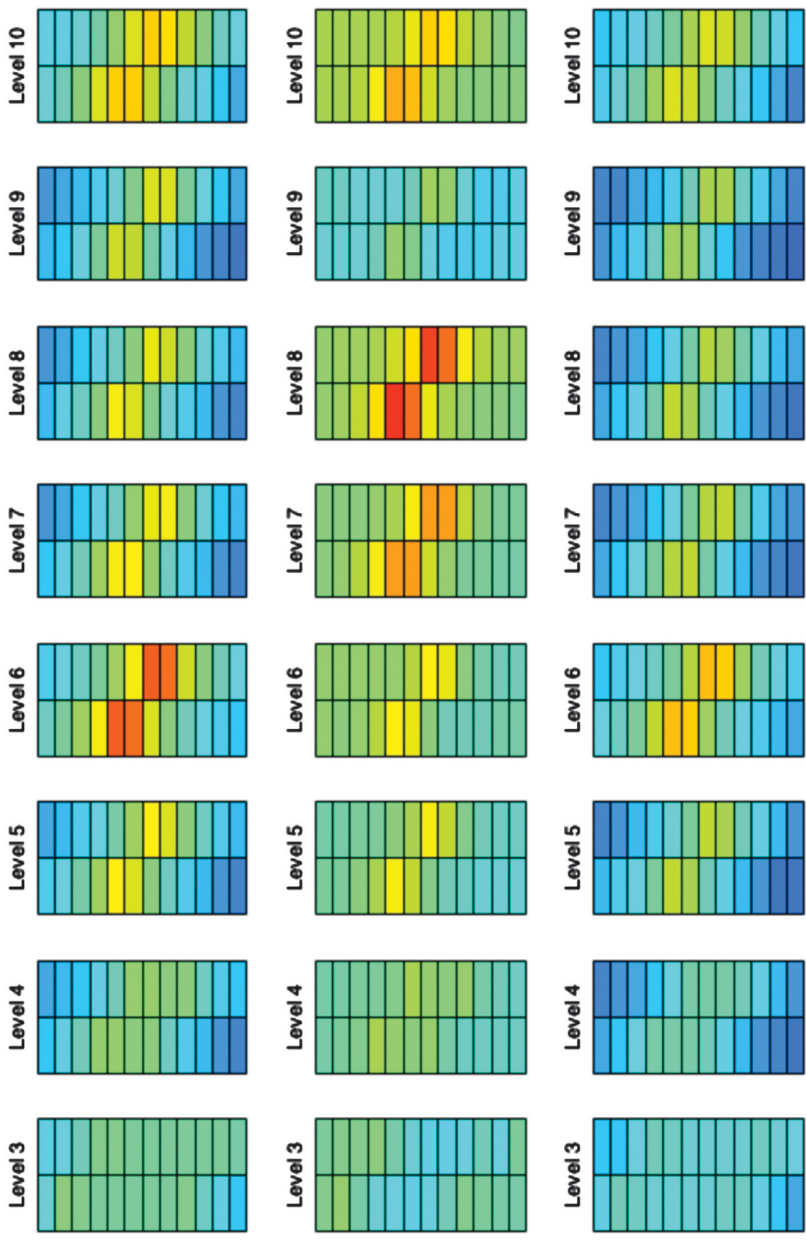

Damage scenario

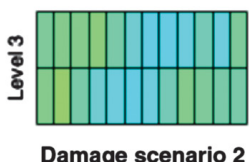

Damage scenario 2
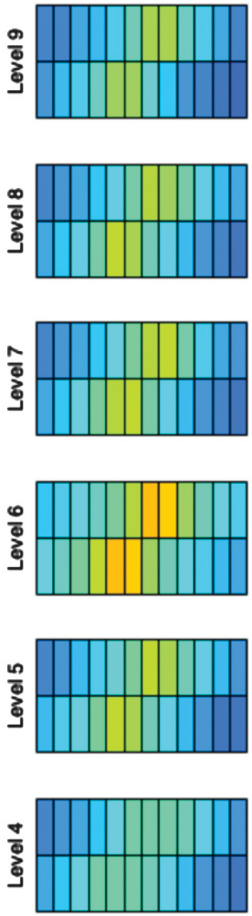

Figure 7. All damage scenarios for the nine-story building, showing each individual template's total correlation over the height of the structure. Each cell represents the damage location on the north and south side of the structure (see Fig. 5) Note that the 10th level is the roof.

localize damage in buildings after strong ground shaking. There are, however, a number of additional analyses that need to be conducted before the method can be practically implemented. The first is to more rigorously connect the im-

Table 2

Damage Scenarios Applied to the Nine-Story Building between Floors Six and Seven

\begin{tabular}{cc}
\hline Scenario & \multicolumn{1}{c}{ Damage Applied } \\
\hline 1 & Location S6 in the $X$ direction \\
2 & Location S6 in the $Y$ direction \\
3 & Location S3 in the $X$ direction \\
4 & Location S3 in the $Y$ direction
\end{tabular}

Note that each damage scenario corresponds to a release of a particular moment connection (converting it to pinned). The $X$ direction corresponds to east-west, and the $Y$ direction corresponds to north-south. See Figure 6 for S3 and S6 damage locations.
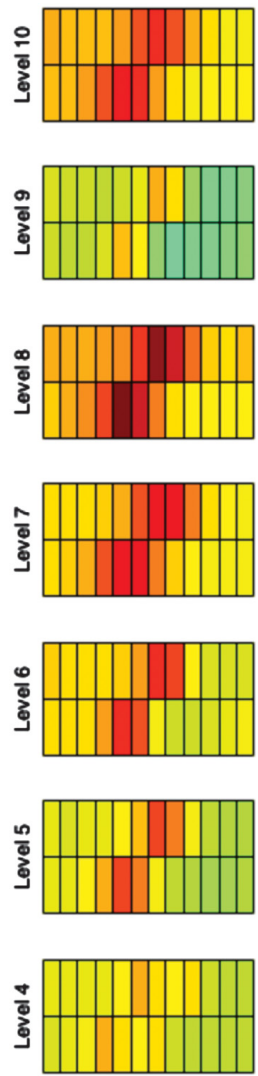

pulsive sources used here to generate the templates for actual damage. This initial test uses changes in effective impedance as the proxy for damage, but with a tighter coupling to the actual element-failure mode, the method might be able to more accurately quantify the damage level for a given correlation value in the template match. Various forms of failure (i.e., buckling and weld failure) will have different scattering properties.

Second, the number and distribution of sensors will determine the resolution and minimum level of detection. Generally, more sensors will decrease the effect of ambient noise on the correlation. Placement of sensors relative to critical structural components (or building contents) will also have an effect on detection levels. A more random deployment, rather than the very regular one used in the two buildings presented here, may be more optimal.

The third area that will need more study is the differencing of pre-event and postevent recordings to enhance the scattered waves. At a minimum, the data from events will need to be deconvolved by the response at the lowest floor in the building to remove, as much as possible, the source-specific effects. This would remove this sensor from participation in the correlations.

\section{Conclusions}

A method for using predetermined templates to isolate damage location and occurrence in a building structure has been presented. Template generation using finite-element models at predetermined locations of potential damage and nonlinearity is an easily implementable strategy. Storing these templates and then using them in densely instrumented structures offers an opportunity to assess the performance of a structure in a new way that can prove valuable to an owner in a postevent status. The correlations described here are not computationally time consuming and could be computed continuously to provide general monitoring of the building.

\section{Data and Resources}

The data used in this study were synthetically created. The modeling was done with ETABS available from Structural and Earthquake Engineering Software, Computers and Structures, Inc. (1995), Berkeley, California, https://www .csiamerica.com/products/etabs and http://docs.csiamerica 
(a)
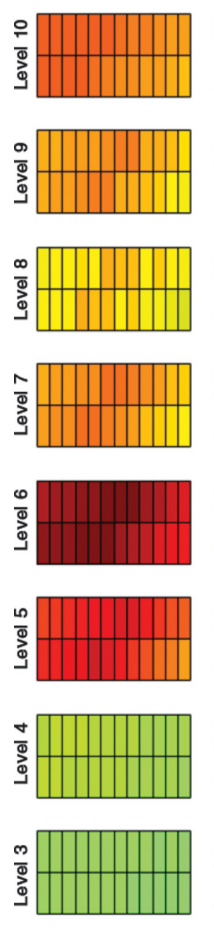

Damage scenario 1
Correlations of $X$-only for all damage scenarios
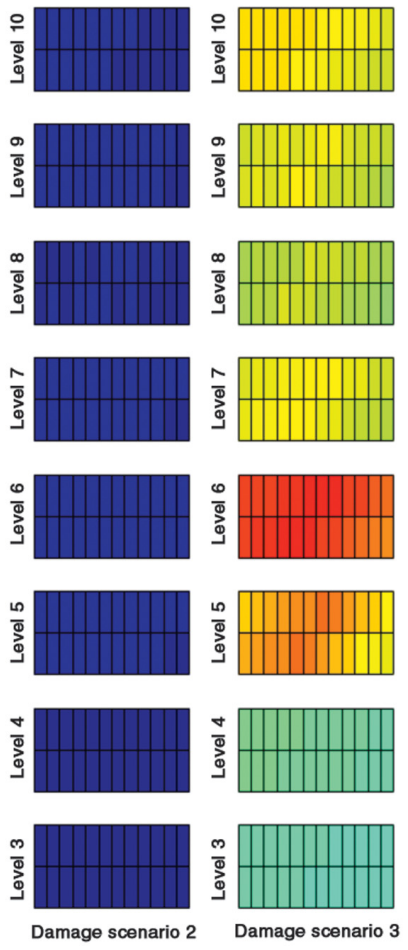
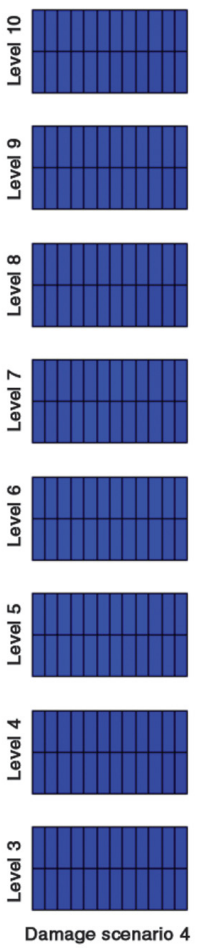

(b) Correlations of $Y$-only for all damage scenarios
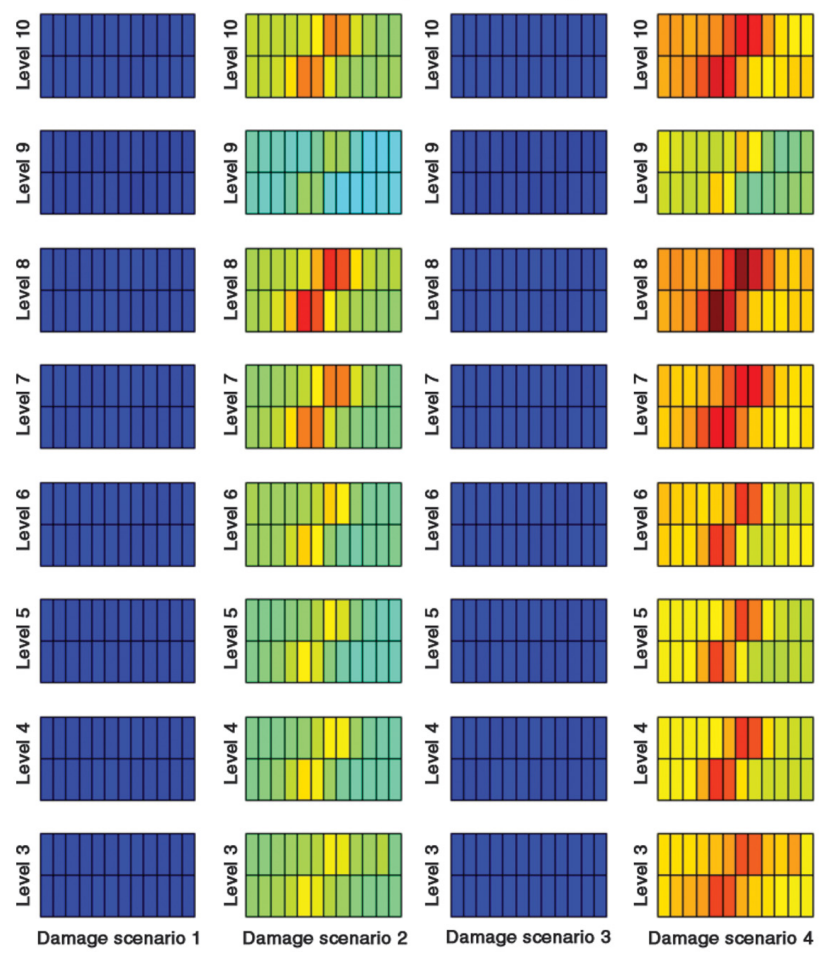

Damage scenario 3

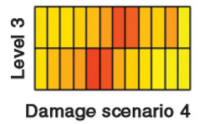

Figure 8. All damage scenarios for the nine-story building, with each individual template's (a) $X$-only and (b) $Y$-only correlation over the height of the structure. The column and row labeling is the same as in Figure 7. Observe that damage in any one direction has the strongest correlation amplitude with the vector component in the same orientation.

.com/manuals/etabs/Analysis\%20Reference.pdf (last accessed August 2018).

\section{Acknowledgments}

The authors thank the Community Seismic Network (CSN) project at Caltech for supplying data for the buildings used in this study and the building owners and engineers for supplying detailed plans that allowed us to build the computational models. A.M. was supported by the Caltech Housner Fellowship during this study.

\section{References}

Celebi, M., H. Ulusoy, and N. Nakata (2016). Responses of a tall building in Los Angeles, California, as inferred from local and distant earthquakes, Earthq. Spectra 32, no. 3, 1821-1843, doi: 10.1193/ 050515EQS065M.

Clayton, R., T. Heaton, M. Kohler, M. Chandy, R. Guy, and J. Bunn (2015). Community seismic network: A dense array to sense earthquake strong motion, Seismol. Res. Lett. 86, no. 5, doi: 10.1785/ 0220150094.

Farrar, C. R., and K. Worden (2007). An introduction to structural health monitoring, Phil. Trans. Roy. Soc. Lond. A 365, no. 1851, 303-315.

Heckman, V. (2014). Damage detection in civil structures using highfrequency seismograms, Ph.D. Dissertation, California Institute of Technology, California, available at http://resolver.caltech.edu/ CaltechTHESIS:12192013-162221707 (last accessed August 2018).

Heckman, V., M. Kohler, and T. Heaton (2011). A damage detection method for instrumented civil structures using prerecorded Green's functions and cross-correlation, Proc. of the 6th International Workshop on
Advanced Smart Materials and Smart Structures Technology, ANCRiSST2011, Dalian, China, 25-26 July.

Huang, Y., and G. Beroza (2015). Temporal variation in the magnitudefrequency distribution during the Guy-Greenbrier earthquake sequence, Geophys. Res. Lett. 42, 6639-6646, doi: 10.1002/2015GL065170.

Kohler, M. D., T. H. Heaton, and M. H. Cheng (2013). The community seismic network and quake-catcher network: Enabling structural health monitoring through instrumentation by community participants, Proc. SPIE 8692, Sensors and Smart Structures Technologies for Civil, Mechanical, and Aerospace Systems, San Diego, California, 19 April 2013, doi: 10.1117/12.2010306.

Kohler, M. D., T. H. Heaton, M. H. Cheng, and P. Singh (2014). Structural health monitoring through dense instrumentation by community participants: The community seismic network and quake-catcher network, 10th U.S. National Conference on Earthquake Engineering (10NCEE), Anchorage, Alaska, 21-25 July 2014.

Kohler, M. D., A. Massari, T. H. Heaton, H. Kanamori, E. Hauksson, R. Guy, R. W. Clayton, J. Bunn, and K. M. Chandy (2016). Downtown Los Angeles 52-story high-rise and free-field response to an oil refinery explosion, Earthq. Spectra 32, no. 3, 1793-1820.

Miller, D. K. (1998). Lessons learned from the Northridge earthquake, Eng. Struct. 20, nos. 4/6, 249-260.

Mordret, A., H. Sun, G. Prieto, N. Toksoz, and O. Buyukoztuk (2017). Continuous monitoring of high-rise buildings using seismic interferometry, Bull. Seismol. Soc. Am. 107, no. 6, 2759-2773, doi: 10.1785/0120160282.

Qiao, L., and A. Esmaeily (2011). An overview of signal-based damage detection methods, Appl. Mech. Mater. 94, 834-851, doi: 10.4028/ www.scientific.net/AMM.94-96.834.

Shelly, D., G. Beroza, and S. Ide (2007). Non-volcanic tremor and low-frequency earthquake swarms, Nature 446, 305-307, doi: 10.1038/ nature05666. 
Todorovska, M., and M. Trifunac (2007). Damage detection in the Imperial County Services Building I: The data and time-frequency analysis, Soil Dynam. Earthq. Eng. 27, 564-576.

Todorovska, M., and M. Trifunac (2008). Damage detection in the Imperial County Services Building III: Analysis of wave travel times via impulse response functions, Soil Dynam. Earthq. Eng. 28, 387-404.

Wen, W., and E. Kalkan (2017). System identification based on deconvolution and cross-correlation: An application to a 20-story instrumented building in Anchorage, Alaska, Bull. Seismol Soc. Am. 107, no. 2, 718-740, doi: 10.1785/0120160069.

Wood, J. H. (1972). Analysis of the earthquake response of a nine-story steel frame building during the San Fernando earthquake, Caltech Earthquake Engineering Research Laboratory, Rept. No. 72-04.

Yoon, C., O. O'Reilly, K. Bergen, and G. Beroza (2015). Earthquake detection though computationally efficient similarity search, Sci. Adv. 1, no. 11, e1501057, doi: 10.1126/sciadv.1501057.
Civil and Mechanical Engineering

California Institute of Technology

MS 104-44

Pasadena, California 91125

kohler@caltech.edu

(A.M., M.K.)

Seismological Laboratory

California Institute of Technology

MS 252-21

Pasadena, California 91125

clay@gps.caltech.edu

(R.W.C.) 